\title{
Background field Landau mode operators for the nucleon
}

\author{
Waseem Kamleh ${ }^{1, \star}$ Ryan Bignell ${ }^{1}$, Derek B. Leinweber ${ }^{1}$, and Matthias Burkardt ${ }^{2}$, \\ ${ }^{1}$ Special Research Centre for the Subatomic Structure of Matter, University of Adelaide, Australia \\ ${ }^{2}$ Department of Physics, New Mexico State University, Las Cruces, NM 88003-001, U.S.A.
}

\begin{abstract}
The introduction of a uniform background magnetic field breaks threedimensional spatial symmetry for a charged particle and introduces Landau mode effects. Standard quark operators are inefficient at isolating the nucleon correlation function at nontrivial field strengths. We introduce novel quark operators constructed from the twodimensional Laplacian eigenmodes that describe a charged particle on a finite lattice. These eigenmode-projected quark operators provide enhanced precision for calculating nucleon energy shifts in a magnetic field. Preliminary results are obtained for the neutron and proton magnetic polarisabilities using these methods.
\end{abstract}

\section{Introduction}

In the presence of a magnetic field $\vec{B}$, for small values of the field strength it is possible to form an expansion for the energy $E$ of a baryon as

$$
E(B)=M+\vec{\mu} \cdot \vec{B}+\frac{|q B|}{2 M}-\frac{1}{2} 4 \pi \beta B^{2}+\cdots
$$

The magnetic moment $\vec{\mu}$ appears in the coefficient of the linear term, and the quadratic term contains the magnetic polarisability $\beta$. The term proportional to $|q B|$ corresponds to the lowest Landau level energy, and is only present for charged hadrons.

The magnetic moments and polarisabilities of the proton and neutron are of great interest, and lattice QCD calculations provide an opportunity to compare with experimental values and gain insight into the underlying physics [1-3]. In particular, experimental measurements of nucleon magnetic polarisabilities remain challenging with considerable uncertainties. Improvement has been seen in recent years [4], providing scope for lattice QCD to make important predictions regarding these values. However, performing a precise lattice calculation of baryon magnetic polarisabilities presents its own challenges. In principle, the method is simple. We can impose a uniform background magnetic field on a lattice gauge field ensemble at a variety of (small) field strengths [5-8] and fit the resulting energies as a function of field strength to extract the magnetic moment and polarisability $[9,10]$.

In practice, due to the overlap of intermediate multi-pion states, baryon correlation functions inherently suffer from a rapidly decaying signal-to-noise problem. This makes the extraction of the magnetic polarisability numerically difficult as it appears at second order in the energy expansion. Previous work studying the magnetic properties of the neutron using the background field method

^ Speaker, e-mail: waseem.kamleh@adelaide.edu.au 


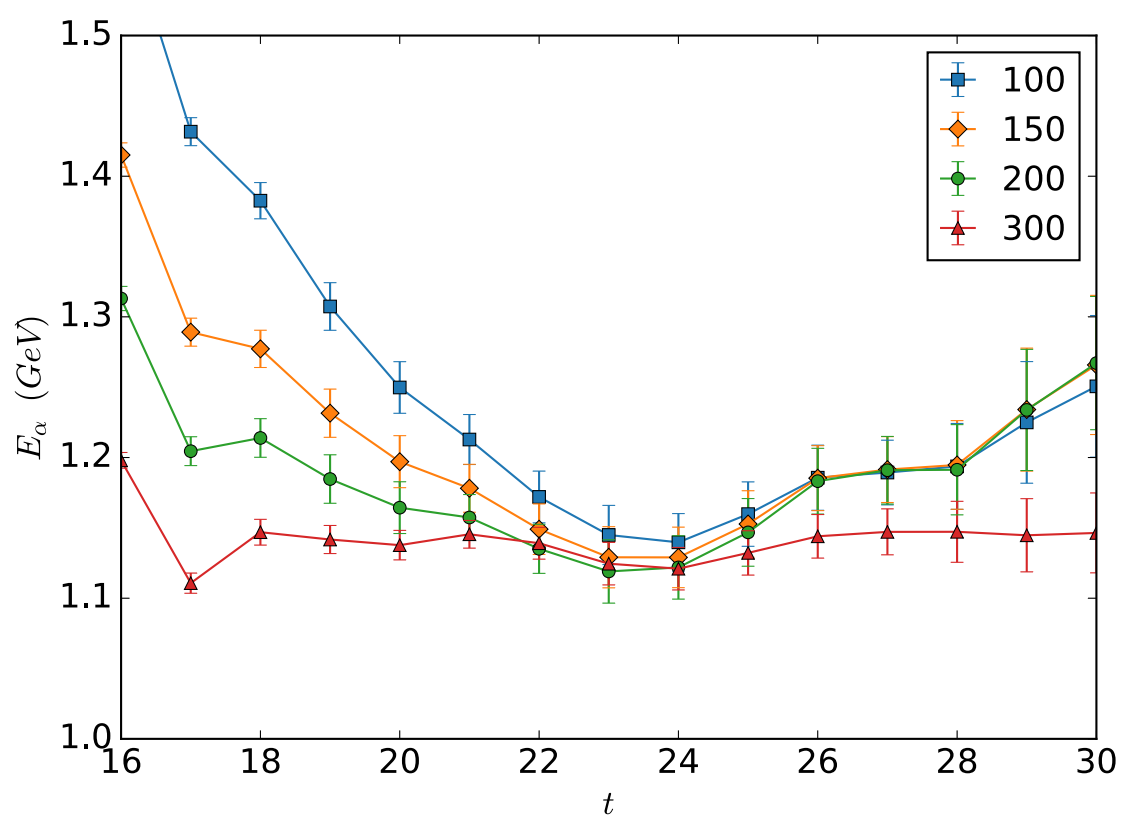

Figure 1. The nucleon effective mass function (at zero magnetic field strength) for various levels of gauge covariant Gaussian smearing at the source, with a point sink.

on the lattice with standard nucleon interpolating fields has demonstrated the difficulty in obtaining a precise estimate of the magnetic polarisability $[8,11]$.

In standard lattice QCD calculations, the use of three-dimensional gauge covariant Gaussian smearing on the quark fields at the source and/or sink is highly effective at isolating the nucleon ground state. However, the imposition of a uniform background field fundamentally alters the physics that is present. The three dimensional spatial symmetry is broken by the magnetic field, and the dynamics of QCD are perturbed by the electromagnetic interactions experienced by the charged quarks.

In the absence of QCD interactions, under the influence of a uniform magnetic field each individual quark will have an associated Landau energy proportional to its charge. If we then turn on QCD interactions the quarks will hadronise, such that in the confining phase the Landau energy corresponds to that of the composite particle. In particular, when forming a neutron, the $d d u$ quarks must combine such that the overall Landau energy vanishes, as there is zero net hadronic charge. Hence, we see that in the confining phase the QCD and magnetic interactions compete with each other. This leads us to the idea of using quark operators on the lattice that capture both of these forces.

In this work we utilise the freedom of choosing asymmetric source and sink operators to construct correlation functions that provide better overlap with the energy eigenstates of the nucleon in a background magnetic field. At the source, we use 300 sweeps of standard Gaussian-smearing, tuned to maximise overlap with the nucleon ground state at $B=0$, as shown in Figure 1. This provides a representation of the QCD interactions. At the sink, we use a projection operator based on the eigenmodes of the two-dimensional lattice Laplacian, in an attempt to capture the physics associated with the magnetic field. 


\section{$2 U(1)$ Landau mode projection}

To construct a uniform magnetic field (in the $z$ direction) on a lattice with spatial extents $\left(n_{x}, n_{y}, n_{z}\right)$, there are multiple gauge-equivalent choices for the potential. We fix the $U(1)$ gauge field to

$$
\begin{aligned}
& U_{1}(x, y)=\exp (-i B y), \\
& U_{2}(x, y)=\left\{\begin{array}{cc}
1, & y<n_{y}-1, \\
\exp \left(+i B n_{y} x\right), & y=n_{y}-1 .
\end{array}\right.
\end{aligned}
$$

Applying the periodic boundary conditions at the corner of the lattice imposes a quantisation condition,

$$
q B a^{2}=\frac{2 \pi k}{n_{x} n_{y}},
$$

where the integer $k$ enumerates the magnetic field strength. For a charged scalar particle the lattice Landau levels correspond to the eigenmodes of the 2D Laplacian,

$$
\Delta_{\vec{x}, \vec{x}^{\prime}}=4 \delta_{\vec{x}, \vec{x}^{\prime}}-\sum_{\mu=1,2}\left(U_{\mu}(\vec{x}) \delta_{\vec{x}+\hat{\mu}, \vec{x}^{\prime}}+U_{\mu}^{\dagger}(\vec{x}-\hat{\mu}) \delta_{\vec{x}-\hat{\mu}, \vec{x}^{\prime}}\right)
$$

While in the continuum, each Landau level has an infinite degeneracy, the degeneracy of the lattice Landau modes is finite and dependent on the magnetic field-strength. Of primary interest is the lowest Landau level on the lattice, which has degeneracy equal to the flux quanta $|k|$.

We first consider the Landau level effects on the lattice as they apply to hadrons, being colour singlet states. A hadron of (integer) charge $q$ experiences a field strength $k_{B}=-3 q k_{d}$ relative to that of the $d$ quark. Consider the momentum-projected hadronic two-point correlator,

$$
G(t, \vec{p})=\sum_{\vec{x}} e^{-i \vec{p} \cdot \vec{x}}\langle\Omega|T\{\chi(t, \vec{x}) \bar{\chi}(0)\}| \Omega\rangle .
$$

Due to the presence of the magnetic field, the energy eigenstates of a charged hadron (such as the proton) in a magnetic field cannot also be eigenstates of the $p_{x}, p_{y}$ momentum components. Instead of performing a 3-dimensional Fourier projection, we can instead project the $x, y$ dependence of $G$ onto the lowest Landau level, and also select a specific value for the $z$ component of momentum,

$$
G\left(t, \vec{B}, p_{z}\right)=\sum_{\vec{x}} \psi_{\vec{B}}(x, y) e^{-i p_{z} z}\langle\Omega|T\{\chi(t, \vec{x}) \bar{\chi}(0)\}| \Omega\rangle .
$$

In the infinite volume case, the lowest Landau mode takes a Gaussian form, $\psi_{\vec{B}}(x, y) \sim e^{-|q B|\left(x^{2}+y^{2}\right) / 4}$. As has been noted elsewhere [10,12], in a finite volume the periodicity alters the form of the wave function. On the lattice, we can calculate the eigenmodes of the 2D Laplacian in Eq. (3) and project the correlator for a charged hadron onto the space spanned by the modes $\psi_{i, \vec{B}}$ associated with the lowest lattice Landau level,

$$
G\left(t, \vec{B}, p_{z}\right)=\sum_{\vec{x}} \sum_{i=1}^{\left|3 q k_{d}\right|} \psi_{i, \vec{B}}(x, y) e^{-i p_{z} z}\langle\Omega|T\{\chi(t, \vec{x}) \bar{\chi}(0)\}| \Omega\rangle .
$$

While this hadronic projection would be applicable to the proton, the neutron has zero charge $q=0$, and does not have hadronic Landau levels. Hence, the energy eigenstates of the neutron can also be eigenstates of $\vec{p}$. However, the neutron is composed of quarks, which due to their charge would have 


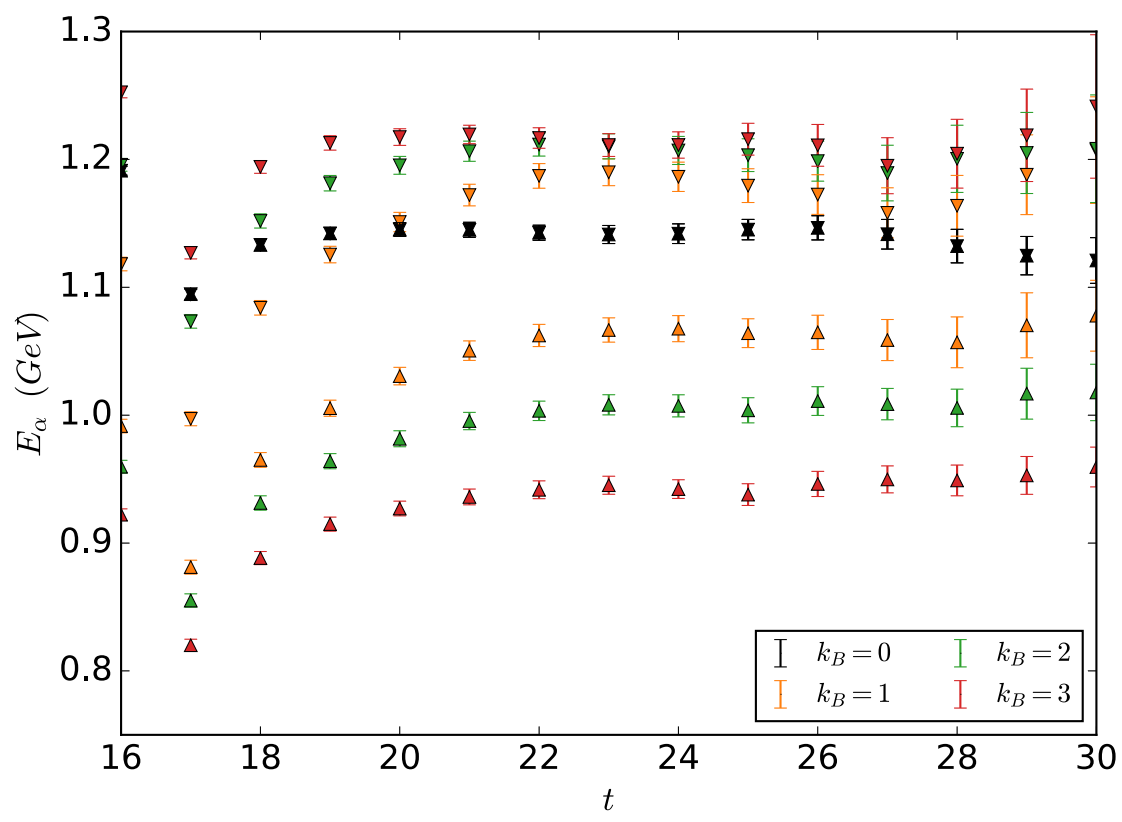

Figure 2. The neutron effective energy function for different magnetic field strengths (enumerated by the integer $k_{B}$ ). Results are shown for both spin polarisations, with the upward/downward pointing triangles corresponding to the spin being aligned/anti-aligned with the magnetic field respectively. We use 300 sweeps of gauge covariant Gaussian smearing at the source. After fixing to Landau gauge, the quark propagators are projected onto the lowest $n$ eigenmodes of the QED-only 2D Laplacian at the sink, where $n$ is equal to $k_{B}$ for the down quark and $2 k_{B}$ for the up quark. We take advantage of the symmetry in the $z$ direction by averaging over both the positive and negative magnetic fields.

individual Landau levels in the absence of QCD interactions. This motivates us to try and capture the Landau mode effects at the quark level. We define a projection operator onto lowest $n$ eigenmodes $\left|\psi_{i, \vec{B}}\right\rangle$ of the 2D Laplacian,

$$
P_{n}=\sum_{i=1}^{n}\left|\psi_{i, \vec{B}}\right\rangle\left\langle\psi_{i, \vec{B}}\right| .
$$

The 2-dimensional projection operator is applied at the sink to the quark propagator.

$$
S_{n}(\vec{x}, t ; \vec{y}, 0)=\sum_{\vec{x}^{\prime}} P_{n}\left(\vec{x}, \vec{x}^{\prime}\right) S\left(\vec{x}^{\prime}, t ; \vec{y}, 0\right) .
$$

where $n=\left|3 q_{f} k_{d}\right|$ modes for the lowest Landau level. As the $U(1)$ Laplacian is not QCD gauge covariant, for the lattice Landau mode projection we fix the gluon field to Landau gauge and apply the appropriate gauge rotation to the quark propagator before projecting. We are free to do this as the hadronic correlation function is gauge invariant, so using a gauge fixed sink operator can only affect the overlap with the ground state, potentially improving the final precision of our result. Figure 2 shows the neutron effective energy function using a smeared source and Landau mode sink for the 


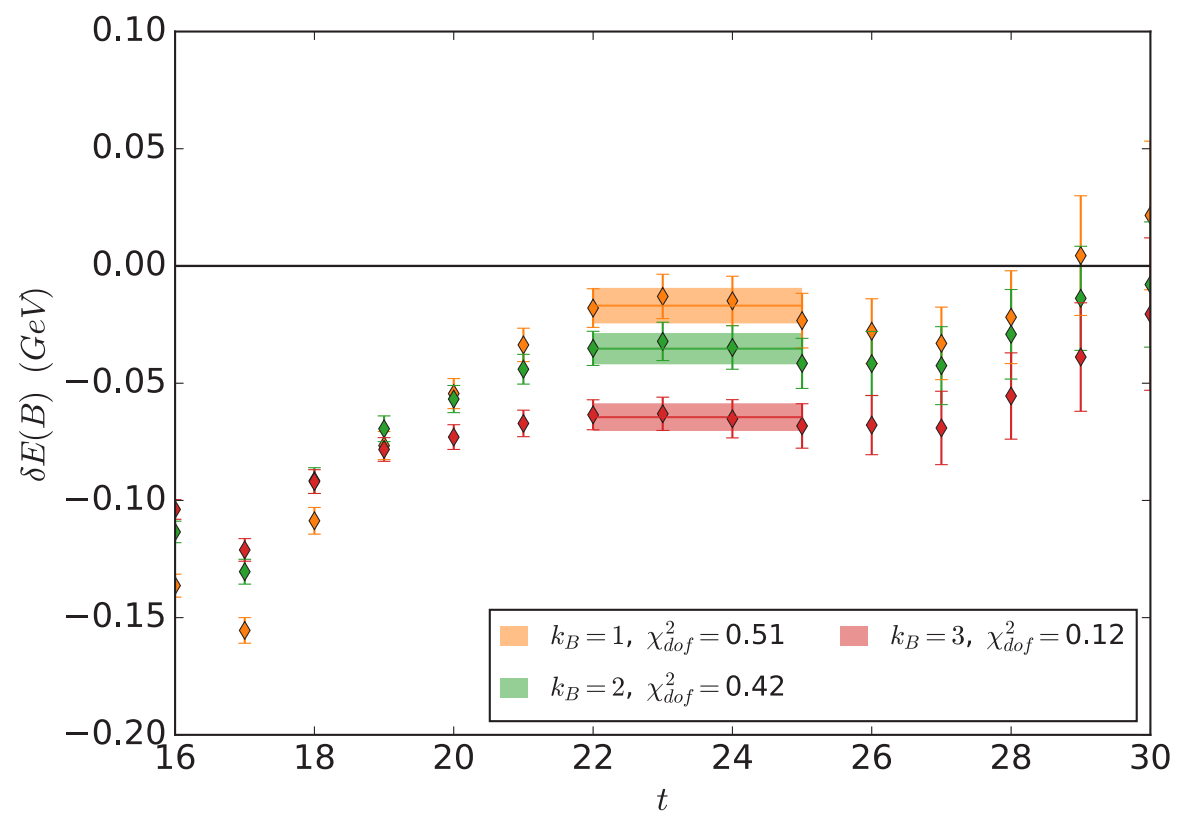

Figure 3. The spin-averaged neutron effective energy shift as a function of Euclidean time (in lattice units), obtained using a smeared source and $U(1)$ Landau mode sink. Results are shown for $k_{B}=1,2,3$ (with magnetic field strength increasing away from zero), along with the selected fits and reduced $\chi^{2}$.

first 3 non-trivial magnetic field strengths, along with the smeared source to point sink correlator at $k_{B}=0$ for comparison. Our results are calculated using the PACS-CS $2+1$ flavour dynamical lattices [13], with $m_{\pi}=413 \mathrm{MeV}$. The calculation is electro-quenched, such that only the valence quarks feel the magnetic field.

In order to obtain the magnetic polarisability, we take advantage of the fact that the magnetic moment term cancels when we average over the two spin polarisations. As the neutron has zero charge and hence no Landau term in the energy expansion (1), the polarisibility becomes the leading term in the spin-averaged energy shift,

$$
\delta E(B)=-\frac{1}{2} 4 \pi \beta B^{2}+O\left(B^{4}\right) .
$$

As the energy shift due to the polarisability is second order in $B$, a precise determination of the energy shift is essential. We obtain the energy shift as a function of field strength $B$ by constructing the spinaveraged neutron correlator and taking the ratio with the zero field $B=0$ correlator. Using this ratio it is possible to reduce the statistical noise in the energy shift, if there are correlated fluctuations that cancel out. We also take advantage of the symmetry in the field direction and average over $\pm B$ fields, such that spin-averaged energy shift correlator $A(B, t)$ at field strength $B$ is given by the following expression

$$
A(B, t)=\left(\frac{G_{\uparrow}(+B, t)+G_{\downarrow}(-B, t)}{G_{\uparrow}(0, t)+G_{\downarrow}(0, t)}\right) \times\left(\frac{G_{\downarrow}(+B, t)+G_{\uparrow}(-B, t)}{G_{\downarrow}(0, t)+G_{\uparrow}(0, t)}\right) .
$$




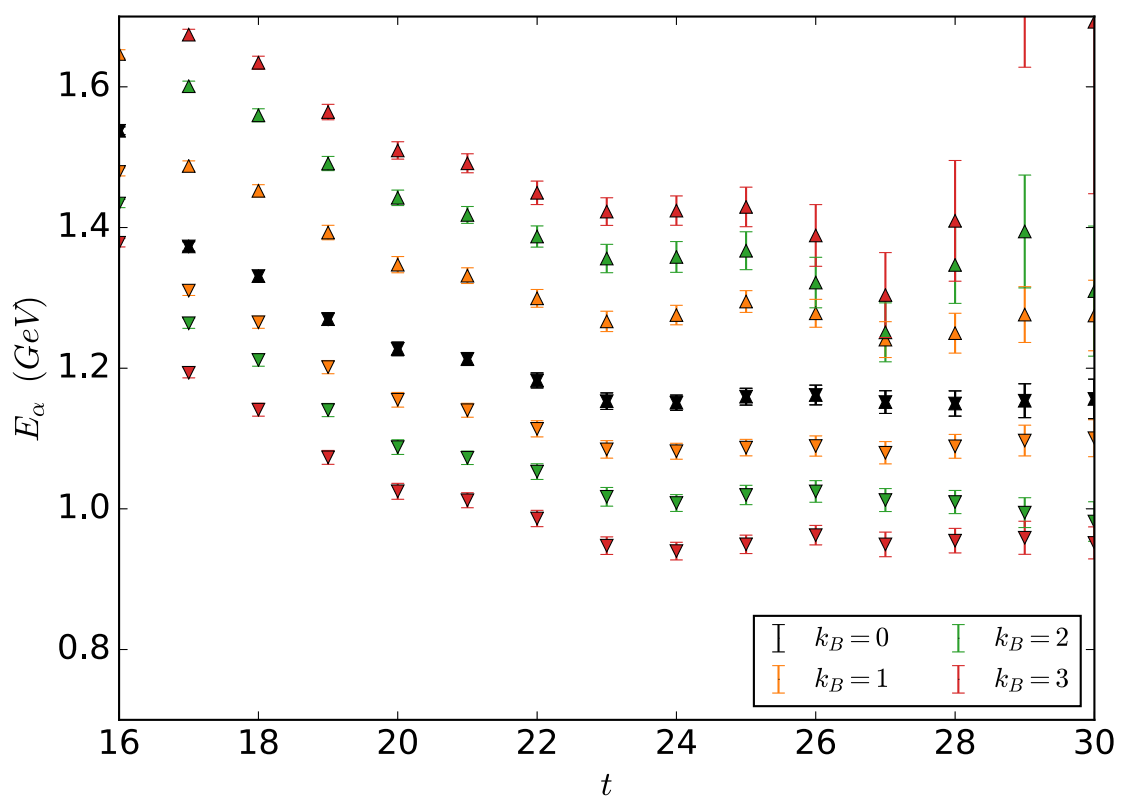

Figure 4. The proton effective energy function for different magnetic field strengths (enumerated by the integer $k_{B}$ ). Results are shown for both spin polarisations, with the upward/downward pointing triangles corresponding to the spin being aligned/anti-aligned with the magnetic field respectively. We use 100 sweeps of gauge covariant Gaussian smearing at the source. The up and down quark propagators are projected onto the lowest $n=96$ eigenmodes of the QED+QCD 2D Laplacian at the sink. We take advantage of the symmetry in the $z$ direction by averaging over both the positive and negative magnetic fields.

The energy shift is then obtained in the standard way by fitting a constant to the effective energy function

$$
\delta E(B, t)=\frac{1}{2} \frac{1}{\delta t} \log \frac{A(B, t)}{A(B, t+\delta t)} .
$$

Figure 3 shows our fits for the neutron spin-averaged energy shift with a smeared source and Landau mode sink. For the first time, we are able to obtain clear plateaus to fit this difficult to obtain second-order term. A fit of the resulting energy shifts as a function of $B$ yields a neutron magnetic polarisability value of $\beta_{n}=1.39(15) \times 10^{-4} \mathrm{fm}^{3}$.

\section{$3 U(1) \times S U(3)$ Laplacian eigenmode projection}

We can construct a gauge covariant projection operator by incorporating the $S U(3)$ gauge links in the definition of the 2D Laplacian in Eq. (3). This ensures that the eigenmodes are QCD covariant, so there is no need to gauge fix. The projector $P_{n}$ is then simply a truncation of the completeness relation,

$$
1=\sum_{i}\left|\psi_{i}\right\rangle\left\langle\psi_{i}\right|
$$




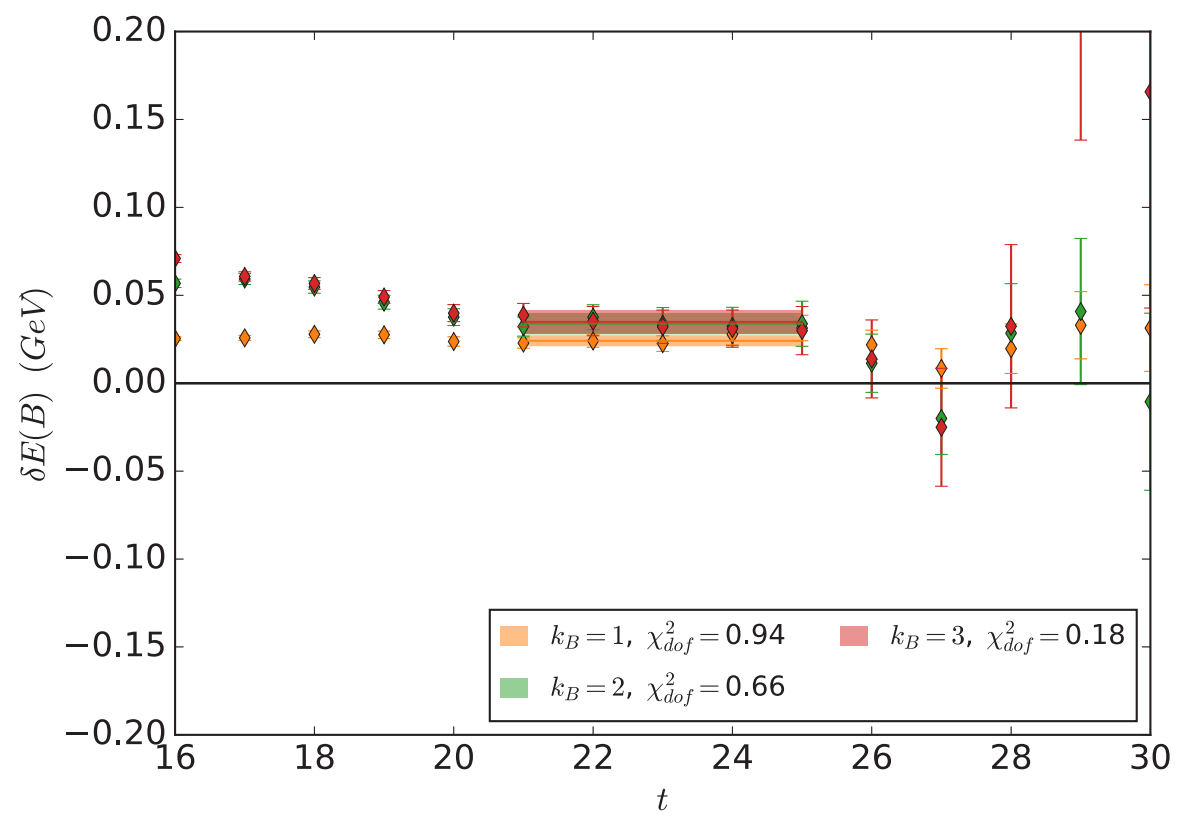

Figure 5. The spin-averaged proton effective energy shift as a function of Euclidean time (in lattice units), obtained using a smeared source and $U(1) \times S U(3)$ eigenmode sink. Results are shown for $k_{B}=1,2,3$ (with magnetic field strength increasing away from zero), along with the selected fits and reduced $\chi^{2}$.

This truncation has a similar effect to performing (2D) smearing, by filtering out the high frequency modes. Indeed, we find that for small values of $n$ the projected hadron correlator becomes noisy, just as it does when performing large amounts of sink smearing.

The introduction of the QCD interactions into the Laplacian causes the $U(1)$ modes associated with the different Landau levels to mix, such that it is no longer possible to clearly identify the modes associated with the lowest Landau level at small field strengths. Instead, we simply choose a fixed number $n>|q k|$ modes to project, where $n$ should be sufficiently large as to avoid introducing large amounts of noise into the two-point correlation function.

An advantage of using the $U(1) \times S U(3)$ Laplacian projector is that it is well defined at zero magnetic field strength, where the $U(1)$ field is equal to unity. This means that the fluctuations at finite $B$ and $B=0$ are strongly correlated, such that they cancel out when taking the ratio of the correlators in Eq. (10), providing an improved signal in comparison to the $U(1)$ projection. This improvement does come at a computational cost, as the $U(1) \times S U(3)$ Laplacian eigenmodes must be calculated on every configuration, whereas the pure $U(1)$ Laplacian eigenmodes need only be calculated once.

As the proton is charged, in addition to the quark level $U(1) \times S U(3)$ Laplacian projection applied to the quark propagator, we also project the proton correlation function onto the lowest hadronic Landau level using Eq. (6). Furthermore, the spin-averaged proton energy shift must now include the hadronic Landau energy term along with the second order polarisability term,

$$
\delta E(B)=\frac{|q B|}{2 M}-\frac{1}{2} 4 \pi \beta B^{2}+O\left(B^{4}\right) .
$$


Figure 5 shows our fits for the proton spin-averaged energy shift with a smeared source and $U(1) \times$ $S U$ (3) Laplacian eigenmode sink. Due to the Landau and polarisibility terms having opposite sign, the variation in the energy shift as a function of field strength is much smaller than for the neutron. Nonetheless, we are again able to obtain clear plateaus, enabling us to extract the energy shifts and fit them as a function of field strength $B$, providing a preliminary result for the proton magnetic polarisability of $\beta_{p}=1.15(24) \times 10^{-4} \mathrm{fm}^{3}$.

\section{Summary}

We have studied the energy shifts of the neutron and proton induced by a magnetic field using a smeared source and two different Laplacian eigenmode projectors at the sink. For the neutron, gauge fixing the quark propagators and projecting on the lowest Landau level using $U(1)$ eigenmodes provides us with clear plateaus for the spin-averaging energy shifts. For the proton, similar success is obtained by a gauge covariant projector formed from the eigenmodes of the $U(1) \times S U(3)$ Laplacian.

It is worthwhile to consider for a moment the physics that underpins the success of our Laplacian eigenmode projection methods. As we are interested in obtaining the magnetic polarisability, we want to work with small $\vec{B}$ field strengths for the perturbative energy expansion to be valid; hence we are in the confined phase of QCD. Confined quarks cannot have individual Landau levels. Nonetheless, the effects of the magnetic field on the quark distribution in the nucleon appear to be significant. A possible explanation for this may be linked to the chiral magnetic effect, induced by the interplay between the magnetic field and the local gauge field topology. Indeed, based on topological arguments in two-dimensions, a recent staggered quark study at finite temperature finds that the contribution of the lowest Landau level eigenmodes remains important even after QCD interactions are introduced [14].

Based on the success of the methods presented herein, we are now in a position to provide precise determinations of the nucleon magnetic polarisabilities using lattice QCD, and our calculations will be published in full elsewhere [15].

\section{References}

[1] K. Kossert et al., Phys. Rev. Lett. 88, 162301 (2002), nucl-ex/0201015

[2] H.W. Griesshammer, J.A. McGovern, D.R. Phillips, G. Feldman, Prog. Part. Nucl. Phys. 67, 841 (2012), 1203.6834

[3] P. Wang, D.B. Leinweber, A.W. Thomas, Phys. Rev. D89, 033008 (2014), 1312 . 3375

[4] L.S. Myers et al. (COMPTON@MAX-lab), Phys. Rev. Lett. 113, 262506 (2014), 1409. 3705

[5] J. Smit, J.C. Vink, Nucl. Phys. B286, 485 (1987)

[6] C.W. Bernard, T. Draper, K. Olynyk, M. Rushton, Phys. Rev. Lett. 49, 1076 (1982)

[7] M. Burkardt, D.B. Leinweber, X.m. Jin, Phys. Lett. B385, 52 (1996), hep-ph/9604450

[8] E. Chang, W. Detmold, K. Orginos, A. Parreno, M.J. Savage, B.C. Tiburzi, S.R. Beane (NPLQCD), Phys. Rev. D92, 114502 (2015), 1506.05518

[9] G. Martinelli, G. Parisi, R. Petronzio, F. Rapuano, Phys. Lett. B116, 434 (1982)

[10] B.C. Tiburzi, S.O. Vayl, Phys. Rev. D87, 054507 (2013), 1210. 4464

[11] T. Primer, W. Kamleh, D. Leinweber, M. Burkardt, Phys. Rev. D89, 034508 (2014), 1307.1509

[12] R. Bignell, W. Kamleh, D. Leinweber, M. Burkardt, PoS INPC2016, 287 (2017), 1704.08435

[13] S. Aoki et al. (PACS-CS), Phys. Rev. D79, 034503 (2009), 0807. 1661

[14] F. Bruckmann, G. Endrodi, M. Giordano, S.D. Katz, T.G. Kovacs, F. Pittler, J. Wellnhofer, Phys. Rev. D96, 074506 (2017), 1705.10210

[15] R. Bignell, W. Kamleh, D. Leinweber, M. Burkardt, in preparation. 